\title{
Sequestering carbon dioxide in a closed underground volume
}

\author{
Christine Ehlig-Economides ${ }^{\mathrm{a}, 1}$, Michael J. Economides ${ }^{\mathrm{b}, *}$ \\ a Department of Petroleum Engineering, Texas A\&M University, College Station, Texas 77843, USA \\ b Department of Chemical Engineering, University of Houston, Houston, Texas 77204, USA
}

\section{A R T I C L E I N F O}

\section{Article history:}

Received 11 October 2009

Accepted 4 November 2009

\section{Keywords:}

sequestration

carbon dioxide

greenhouse gas management injectivity

deep saline aquifers

closed system injection

\begin{abstract}
A B S T R A C T
The capture and subsequent geologic sequestration of $\mathrm{CO}_{2}$ has been central to plans for managing $\mathrm{CO}_{2}$ produced by the combustion of fossil fuels. The magnitude of the task is overwhelming in both physical needs and cost, and it entails several components including capture, gathering and injection. The rate of injection per well and the cumulative volume of injection in a particular geologic formation are critical elements of the process.

Published reports on the potential for sequestration fail to address the necessity of storing $\mathrm{CO}_{2}$ in a closed system. Our calculations suggest that the volume of liquid or supercritical $\mathrm{CO}_{2}$ to be disposed cannot exceed more than about $1 \%$ of pore space. This will require from 5 to 20 times more underground reservoir volume than has been envisioned by many, and it renders geologic sequestration of $\mathrm{CO}_{2}$ a profoundly non-feasible option for the management of $\mathrm{CO}_{2}$ emissions.

Material balance modeling shows that $\mathrm{CO}_{2}$ injection in the liquid stage (larger mass) obeys an analog of the single phase, liquid material balance, long-established in the petroleum industry for forecasting undersaturated oil recovery. The total volume that can be stored is a function of the initial reservoir pressure, the fracturing pressure of the formation or an adjoining layer, and $\mathrm{CO}_{2}$ and water compressibility and mobility values.

Further, published injection rates, based on displacement mechanisms assuming open aquifer conditions are totally erroneous because they fail to reconcile the fundamental difference between steady state, where the injection rate is constant, and pseudo-steady state where the injection rate will undergo exponential decline if the injection pressure exceeds an allowable value. A limited aquifer indicates a far larger number of required injection wells for a given mass of $\mathrm{CO}_{2}$ to be sequestered and/or a far larger reservoir volume than the former.
\end{abstract}

(c) 2009 Elsevier B.V. All rights reserved.

\section{Introduction}

According to the United Nations Intergovernmental Panel for Climate Change (IPCC, 2007), "the increases in atmospheric carbon dioxide $\left(\mathrm{CO}_{2}\right)$ and other greenhouse gases during the industrial era are caused by human activities," and the IPCC insists that anthropogenic greenhouse gas emissions are harmful to the planet and are causing global climate change evident as global temperature rise and local weather extremes. Although greenhouse gases include water vapor, carbon dioxide, and methane, that are emitted through various means, the focus of this paper is strictly on carbon dioxide emissions.

In 2008 coal consumption for electric power generation in the United States was 1.04 billion short tons (tonnes) per year (EIA, 2009), and total carbon dioxide emissions in 2007 were 6.02 billion metric tons (tonnes) including 2.16 billion tonnes from coal fired

\footnotetext{
* Corresponding author. Tel.: +1 713 7253826; fax: +1 7137812548.

E-mail addresses: cee@economidesconsultants.com (C. Ehlig-Economides), mje@uh.edu (M.J. Economides).

${ }^{1}$ Tel.: +1 2819483621 ; fax: +1 7137812548 .
}

electric power generation, 2.6 billion tonnes from petroleum consumption mainly for transportation, and 1.2 billion tonnes from natural gas consumption. By 2030 US carbon dioxide emissions are forecast to reach 6.41 billion tonnes according to the EIA. The Kyoto Protocol proposed for the US to reduce carbon dioxide emissions to $93 \%$ of the 1990 emission level, or to keep it at a level below 4.67 billion tonnes for every year from December 1997, the year of its enactment, and onward. To satisfy the Kyoto Protocol, carbon dioxide emissions should already be reduced and would have to be reduced by 1.75 billion tonnes per year by 2030 . This task is enormous and will be exacerbated further by recent legislation that proposes even more stringent goals.

Potential ways to reduce carbon dioxide emissions include reducing the need for fossil fuel combustion through more efficient energy use (although history has not proven this to be successful), substituting biofuel, hydrogen, or electric power for hydrocarbons in the transportation and electric power generation sectors, substituting natural gas for coal in electric power generation, substituting alternative energy sources for coal and natural gas in electric power generation, and capturing and sequestering carbon dioxide produced 
by combustion. While it is probably not feasible to capture and sequester carbon dioxide emitted from the transportation sector, there is considerable interest in the possibility of sequestering carbon dioxide produced from electric power generation. In particular, because new technologies for electric power generation from coal such as integrated gasification combined cycle (IGCC) produce about $90 \%$ of the carbon dioxide in a concentrated stream presumably suitable for underground sequestration, there is interest in carbon capture and sequestration (CCS) for future electric power generation from coal. CCS for retrofitted coal combustion electric power plants and for natural gas combined cycle (NGCC) plants is potentially feasible as well but at much higher cost. The common assumption is that the cost of carbon sequestration is much less than the cost of carbon capture (NETL, 2007). Further, current energy legislation assumes that the cost of power generation with CCS will be competitive with alternative energy options. We are not convinced that the recovery of carbon dioxide from low pressure combustion gas streams will ever be as efficient or effective as some have suggested but this discussion is outside the scope of this paper.

There are several processes that have been postulated as means for carbon dioxide sequestration. These include ocean sequestration that involves either deep release of the gas, causing dissolution in water or by promoting phytoplankton growth causing consumption of carbon dioxide. Other possibilities include mineral and biological sequestration involving the reaction of carbon dioxide with e.g., magnesium silicate. Biological processes may lead to carbonates or methane. Reforestation may also contribute to sequestration as increased vegetation may consume more carbon dioxide. While all these techniques have received attention they all have time constraints and considerable logistical problems. Geological sequestration has been espoused by many and it is the subject of this paper.

If all of the 1.75 billion tonnes annual reduction forecast for 2030 were to be achieved by sequestering carbon dioxide underground, this would amount to injection of 39 million bpd of supercritical carbon dioxide, assuming a density of $47.6 \mathrm{lbm} / \mathrm{ft}^{3}$. The US currently produces crude oil and lease condensate at a rate of about 5.4 million STB/d with actual reservoir volume perhaps slightly greater depending on the average formation volume factor. By comparison, adding current natural gas and natural gas liquid production at 11.8 million barrels of oil equivalent (BOE) per day gives a total US liquid and gaseous hydrocarbon voidage rate of about 16.2 million BOE/d with much of the crude oil production supported by pressure maintenance via waterflooding or an active water drive (www.eia.doe.gov).

As another comparison, the US currently injects about 38 million bpd of oilfield water. Although this may appear to offer a reassuring analogy to the $\mathrm{CO}_{2}$ volume, in reality it is not, because oilfield water is typically injected in hydraulic communication with the oil or gas production to achieve pressure maintenance and thereby avoid surface subsidence that can occur from underground pore pressure depletion. Injected water usually replaces fluids that are produced and, still, water breakthrough is a common occurrence. Likewise, industrial, municipal, and agricultural groundwater use is strictly monitored, and optimal water management restricts groundwater use to what is recharged via annual precipitation. Both oilfield water injection and groundwater production are, thus, largely steady state processes.

In contrast, carbon dioxide sequestration is not generally envisioned to be associated with any production of underground fluids, and analogies between carbon dioxide sequestration in deep saline aquifers or in depleted hydrocarbon reservoirs and EOR displacement processes are highly inappropriate.

In volumetric terms, for coal density of $94 \mathrm{lbm} / \mathrm{ft}^{3}$ (depends on the type of coal) and supercritical carbon dioxide density of $48 \mathrm{lbm} / \mathrm{ft}^{3}$ (depends on pressure and temperature), more than twice the volume is required to sequester carbon dioxide underground than to remove carbon as coal. However, while a coal seam is approximately $100 \%$ coal, the carbon dioxide must be injected into rock with porosity on the order of $20 \%$, representing 10 times more volume than originally occupied underground by the coal. Further, this paper will show that the volume multiplier is another 50 times more when compressibility and solubility are taken into account. The net result is that it takes more than 500 times more volume to sequester carbon dioxide than was originally occupied as coal. The pore volume required to sequester 1.75 billion tonnes is 182 billion barrels annually, and this represents about 8.5 times the total US crude oil reserves of about 21.5 billion barrels.

To demonstrate these claims, this paper will consider carbon dioxide sequestration via EOR, in deep saline aquifers, and in depleted hydrocarbon reservoirs, using as a basis the emissions from an average coal power plant with generating capacity of $500 \mathrm{MW}$. Our very sobering conclusion is that underground carbon dioxide sequestration via bulk $\mathrm{CO}_{2}$ injection is not feasible at any cost.

\section{Geologic sequestration methods}

While other potential mechanisms for carbon dioxide sequestration may be under consideration, petroleum engineers offer the most expertise for sequestration in an underground porous medium. This section considers two approaches: 1) via EOR or 2) via bulk carbon dioxide injection into a depleted oil or gas reservoir or a deep saline aquifer.

\subsection{EOR}

Oil recovery can often be enhanced by carbon dioxide injection, and this approach has been used commercially for many decades. Traditionally EOR follows waterflooding, and the enhanced oil recovery factor is typically a small fraction of the oil in place. With total (not annual) US oil reserves currently estimated by EIA at 21.5 billion barrels, if even $10 \%$ of this could be enhanced via carbon dioxide injection, the amount would represent on the order of 2 billion barrels, which would represent just under $14 \%$ of the Kyoto Protocol target of 1.75 billion tonnes (14.4 trillion barrels) for annual (not total) carbon dioxide reduction. The current worldwide use of $\mathrm{CO}_{2}$ for EOR is about 57 million tonnes per year, about 3\% of just the US mandated Kyoto Protocol reduction (Evans and Melzer, 2009).

\subsection{Bulk carbon dioxide injection}

The most commonly recommended method for carbon dioxide sequestration is by bulk injection into a depleted oil or gas reservoir or a deep saline aquifer. For depleted oil reservoirs, it is important to consider by what mechanism depletion occurred before field abandonment. If the field was abandoned following primary oil recovery only without active water drive, the average reservoir pressure may be considerably below the original reservoir pressure. In contrast, if the field was produced under active water drive or under waterflood, the abandonment pressure may be 
at approximately the original reservoir pressure or approximately the original bubble point pressure. In all cases the pore space is likely to be saturated mainly by liquid. Likewise for deep saline aquifers the pore space is saturated by brine. For depleted gas reservoirs, the pore space may be saturated by gas at abandonment pressure well below the original reservoir pressure plus connate water or it may be mainly saturated by water at original reservoir pressure if the gas was produced under active water drive.

By far the best prospect among these choices for bulk carbon dioxide injection is an abandoned gas reservoir depleted without active water drive. However, typically such reservoirs are used for natural gas storage and would not be available for carbon dioxide sequestration. Of the liquid saturated prospects, oil reservoirs abandoned at lower than initial pressure will offer somewhat more storability than oil reservoirs abandoned after waterflood or deep saline aquifers. The following discussion provides a conceptual model for bulk $\mathrm{CO}_{2}$ injection in a deep saline aquifer, and with minor adjustments this would apply to any liquid filled underground reservoir, including depleted oil and gas reservoirs.

There are two considerations: the wellbore pressure increase over average reservoir pressure, and the increase in average reservoir pressure over the initial reservoir pressure. For a deep saline aquifer, the initial formation pressure in psi is likely to be hydrostatic and therefore equal to $0.433 \mathrm{H}$, where $\mathrm{H}$ is the aquifer depth in $\mathrm{ft}$. The formation temperature will be a function of the geothermal gradient, which on average may be on the order of $1^{\circ} \mathrm{F}$ per $100 \mathrm{ft}$. With a critical pressure of $1071 \mathrm{psi}$ and critical temperature of $87.8^{\circ} \mathrm{F}, \mathrm{CO}_{2}$ will be in a supercritical state at bottomhole injection conditions for aquifer depths exceeding $2473 \mathrm{ft}$. This is preferred because supercritical $\mathrm{CO}_{2}$ is denser than gaseous $\mathrm{CO}_{2}$ and, therefore, enables storage of more mass per unit underground pore volume.

At first, the bottomhole pressure during $\mathrm{CO}_{2}$ injection at a constant rate is governed by transient flow of single phase brine given by the following equation:

$$
p_{\mathrm{wi}}=p_{\mathrm{i}}-\frac{70.6\left(-q_{\mathrm{CO}_{2}}\right) \mu_{\mathrm{w}}}{k h} \ln \left(\frac{k t}{1688 \phi \mu c_{\mathrm{ti}} r_{\mathrm{w}}^{2}}\right)
$$

where the downhole injection rate is shown as $-q_{\mathrm{CO}_{2}}$, in bpd; wellbore injection and initial reservoir pressures are $p_{\mathrm{wi}}$ and $p_{\mathrm{i}}$, both in psi; $t$ in hours, $k$ and $\phi$ are the aquifer absolute permeability in md and porosity; $r_{\mathrm{w}}$ is the well radius in $\mathrm{ft}$; $\mu_{\mathrm{w}}$ is the brine viscosity, and $c_{\mathrm{ti}}$ is the initial total compressibility in $\mathrm{psi}^{-1}$ accounting for brine and rock compressibility at initial injection conditions. During this early injection period, the injection rate may be ramped up gradually to avoid injecting at a pressure above the formation fracture pressure, $p_{\mathrm{f}}$, which depends on the formation fracture gradient, which for almost all reservoirs will range from 0.71 to $0.82 \mathrm{psi} / \mathrm{ft}$ (Economides and Nolte, 2000). After a relatively short period, typically lasting from a few days to a few months, the bulk carbon dioxide injection establishes a zone near the well in which $\mathrm{CO}_{2}$ flows as a single phase zone surrounded by a two-phase region where the saturation varies from nearly $100 \% \mathrm{CO}_{2}$ to $100 \%$ brine according to Buckley and Leverett (1942) displacement theory. Burton et al. (2008) provide equations for the radii of the single phase and two-phase zones and the pressure drop across each of these zones as well as the pressure drop in the single phase brine.

For this study, the pressure increase over average reservoir pressure is given by

$$
p_{\mathrm{wi}}=\bar{p}-\frac{141.2\left(-q_{\mathrm{CO}_{2}}\right)}{k h}\left[\frac{\mu_{\mathrm{CO}_{2}}}{k_{\mathrm{r}, \mathrm{C}_{\mathrm{CO}_{2}}=1}} \ln \left(\frac{r_{\mathrm{dry}}}{r_{\mathrm{w}}}\right)+\left.\left(\frac{k_{\mathrm{CO}_{2}}}{\mu_{\mathrm{CO}_{2}}}+\frac{k_{\mathrm{rw}}}{\mu_{\mathrm{w}}}\right)^{-1}\right|_{\mathrm{SO}_{2} \text {, avg }} \ln \left(\frac{r_{\mathrm{BL}}}{r_{\mathrm{dry}}}\right)+\mu_{\mathrm{w}} \ln \left(\frac{0.472 r_{\mathrm{e}}}{r_{\mathrm{BL}}}\right)\right]
$$

where $\mathrm{CO}_{2}$ and water viscosities are $\mu_{\mathrm{CO}_{2}}$ and $\mu_{\mathrm{w}}$ in cp; relative permeabilities are $k_{\mathrm{CO}_{2}}$ and $k_{\mathrm{rw}}$; and outer radii of the single phase $\mathrm{CO}_{2}$, 2-phase Buckley-Leverett, and single phase brine are $r_{\mathrm{dry}}, r_{\mathrm{BL}}$, and $r_{\mathrm{e}}$. The relative permeability of the $\mathrm{CO}_{2}$ in the single phase region is $k_{\mathrm{r}, S_{\mathrm{CO} 2}=1}$, and relative permeability values in the 2-phase region are evaluated at the average $\mathrm{CO}_{2}$ saturation according to Buckley-Leverett displacement theory. The factor 0.472 in the last natural logarithm term in Eq. (2) accounts for average reservoir pressure, $\bar{p}$, as the average pressure in the brine region and is a departure from the Burton et al. (2008) approach, which claimed, incorrectly, that treating the aquifer as open, with a constant pressure outer boundary, was equivalent to modeling an effectively infinite aquifer.

Eq. (2) assumes the aquifer volume is limited and that pseudo-steady state flow behavior is established. The open aquifer, or steady state, flow condition assumes that at some distance, pressure in the aquifer is held at a constant value. For this to be true in practice, the aquifer must either outcrop to the land surface or in a stream, lake, or ocean bed where it would be in equilibrium either with atmospheric pressure or with the pressure at the stream, lake or ocean bottom. An outcropping aquifer would provide a potential path for injected $\mathrm{CO}_{2}$ to escape back to the atmosphere, thereby defeating the purpose of $\mathrm{CO}_{2}$ sequestration.

The consequence of assuming the aquifer has a limited area is that the average aquifer pressure will increase over time. Thus, accounting for material balance,

$$
\left(\bar{p}-p_{\mathrm{i}}\right) V_{\mathrm{r}} c_{\mathrm{t}}=V_{\mathrm{CO}_{2}}
$$

where $V_{\mathrm{CO}_{2}}$ is the total volume of $\mathrm{CO}_{2}$ to be injected over the life of the sequestration project, $V_{\mathrm{r}}$ is the minimum required aquifer pore volume to store this volume of $\mathrm{CO}_{2}$, and $c_{t}$ is the total compressibility accounting for $\mathrm{CO}_{2}$, brine, and rock compressibility as

$$
c_{\mathrm{t}}=\frac{\left[\left(r_{\mathrm{dry}}^{2}-r_{\mathrm{w}}^{2}\right) c_{\mathrm{CO}_{2}}+\left(r_{\mathrm{dry}}^{2}-r_{\mathrm{BL}}^{2}\right)\left[S_{\mathrm{CO}_{2}, \mathrm{avg}} c_{\mathrm{CO}_{2}}+\left(1-S_{\mathrm{CO}_{2}, \mathrm{avg}}\right)\right]+\left(r_{\mathrm{e}}^{2}-r_{\mathrm{BL}}^{2}\right) c_{\mathrm{w}}\right]}{\left(r_{\mathrm{e}}^{2}-r_{\mathrm{w}}^{2}\right)}+c_{\mathrm{f}}
$$

using a bulk volume weighted average.

Finally, the difference between the wellbore injection pressure and the initial reservoir pressure will be

$$
p_{\mathrm{wi}}-p_{\mathrm{i}}=p_{\mathrm{wi}}-\bar{p}+\bar{p}-p_{\mathrm{i}}=-\frac{141.2\left(q_{\mathrm{CO}_{2}}\right)}{k h}\left[\frac{\mu_{\mathrm{CO}_{2}}}{k_{r, \mathrm{~S}_{\mathrm{CO}_{2}}}=1} \ln \left(\frac{r_{\mathrm{dry}}}{r_{\mathrm{w}}}\right)+\left.\left(\frac{k_{\mathrm{CO}_{2}}}{\mu_{\mathrm{CO}_{2}}}+\frac{k_{\mathrm{rw}}}{\mu_{\mathrm{w}}}\right)^{-1}\right|_{\mathrm{SO}_{2}, \text { avg }} \ln \left(\frac{r_{\mathrm{BL}}}{r_{\mathrm{dry}}}\right)+\mu_{\mathrm{w}} \ln \left(\frac{0.472 r_{\mathrm{e}}}{r_{\mathrm{BL}}}\right)\right]+\frac{V_{\mathrm{CO}_{2}}}{V_{\mathrm{r}} \mathrm{c}_{\mathrm{t}}}
$$

Many of the published works seem to be consumed by simulating the physics and thermodynamics of $\mathrm{CO}_{2}$ displacing brine or its dissolution in the brine (the latter is a woefully slow process), while they are missing by far the most fundamental issue: during injection sequestration is not displacement but permanent storage in a closed system. Several authors (Kumar et al., 2005; Baklid and Korbo, 1996; Pruess, 2004; Nghiem et al., 
2004; Sengul, 2006; Izpec et al., 2006) employ a constant pressure outer boundary when modeling $\mathrm{CO}_{2}$ injection, which is convenient, but misleading. Actually, flow behavior in a reservoir with a constant pressure boundary does not mimic that of an effectively infinite aquifer, and authors who employ this condition are significantly misrepresenting this case. Likewise, authors like Orr (2004) and Noh et al. (2004), who emphasize the analogies with EOR, are on the wrong track. The consequence of these misrepresentations is that the volume required for $\mathrm{CO}_{2}$ storage has been severely underestimated.

Pruess et al. (2001) modeled $\mathrm{CO}_{2}$ injection in an infinite aquifer, but their approach again significantly overestimated storability. To their credit, van Engelenburg and Blok (1993), Schembre-McCabe et al. (2007), van der Meer and van Wees (2006), Ennis-King and Paterson (2002), and House et al. (2003), have already tried to alert investigators to the issue of pressure buildup in a limited aquifer, and Zakrisson et al. (2008) specifically address modeling multiple injection wells.

There are already some data that seem to warn of problems in the very few existing injection projects. The much cited Sleipner reservoir in the North Sea, as a successful case of $\mathrm{CO}_{2}$ injection (about 1 million tonnes per year compared to 3 million that would be required for a 500 MW coal power plant) shows that much less $\mathrm{CO}_{2}$ is stored radially than what seismic reflection data show (Bickle et al., 2007). They have seen significant leakage to overlying layers. The far reduced radial volume was attributed by the authors to the "significantly reduced... relative permeability of $\mathrm{CO}_{2}$ ". They did not attempt to model the reservoir pressure profile.

\subsection{Application for a single power plant}

A modern commercial $500 \mathrm{MW}$ coal power plant generates about 3 million metric tons of $\mathrm{CO}_{2}$ per year. Assuming it is captured as a pure $\mathrm{CO}_{2}$ stream, what will be the aquifer pore volume required to store the $\mathrm{CO}_{2}$, and how many wells will be needed if the plant life is assumed to be 30 years?

Suppose an aquifer exists in the vicinity of the plant with porosity $20 \%$, permeability $100 \mathrm{md}$, and thickness $100 \mathrm{ft}$. Suppose further that core analysis provides relative permeability curves

$$
\begin{aligned}
& k_{\mathrm{rw}}=\left[1-\left(\frac{S_{\mathrm{CO}_{2}}}{1-S_{\mathrm{wr}}}\right)\right]^{m} \\
& k_{\mathrm{CO}_{2}}=k_{\mathrm{CO}_{2}}^{0}\left[\frac{S_{\mathrm{CO}_{2}}}{1-S_{\mathrm{wr}}}\right]^{n}
\end{aligned}
$$

with $S_{\mathrm{wr}}=0.558, k_{\mathrm{CO}_{2}}^{0}=0.32, m=3$, and $n=3$.

For an aquifer depth of $6000 \mathrm{ft}$ at a temperature of $150{ }^{\circ} \mathrm{F}$ (assuming geothermal gradient of $1{ }^{\circ} \mathrm{F} / 100 \mathrm{ft}$ ) and hydrostatic pressure of about $2598 \mathrm{psi}$, the supercritical fluid density at reservoir conditions will be about $41 \mathrm{lbm} / \mathrm{ft}^{3}$ (Jarrell et al., 2002). At this density the total volume of $\mathrm{CO}_{2}$ to inject in a 30 year period is 4.86 billion cu ft, or 865 million bbl. The volumetric injection rate is 79,000 bpd. To determine the aquifer area required to inject this volume of $\mathrm{CO}_{2}$, it is necessary to decide how much the aquifer will be pressurized above the initial aquifer pressure. Certainly it should not be pressurized above the formation fracture pressure. Assuming the fracture gradient is $0.7 \mathrm{psi} / \mathrm{ft}$, the average reservoir pressure should not exceed 4200 psi. However, in order to inject at a constant rate for 30 years at the end of this time period, the wellbore injection pressure must exceed the average reservoir pressure as in Eq. (2), and this pressure must not exceed 4200 psi.

Experience with natural gas storage indicates that it is not possible to recover all of the stored gas if the reservoir is pressurized well over the initial reservoir pressure. This has been interpreted as an indication that some of the stored gas has leaked out of the reservoir. Exactly the same result may occur for $\mathrm{CO}_{2}$ storage in an aquifer. Therefore, as a first case, assume the aquifer average pressure will not be elevated by more than $100 \mathrm{psi}$ over the initial aquifer pressure. With this assumption Eq. (3) implies the required aquifer pore volume is $7.7 \mathrm{trillion}$ cu $\mathrm{ft}$. For the given aquifer thickness and porosity, the resulting area is $13,800 \mathrm{sq}$ mi. If the injection pressure is allowed to approach the formation fracture pressure, the difference between injection and average pressures is $4200-2598-100=1502 \mathrm{psi}$, and Eq. (2) indicates that $1 / 2$ the required rate can be produced in $1 / 2$ of this area without exceeding this pressure constraint. Therefore, 2 wells can inject all of the $\mathrm{CO}_{2}$ produced by the plant for 30 years.

However, as points of reference, the Prudhoe Bay reservoir area is 337 sq mi, and 9 US states and the District of Columbia all have areas less than 13,800 sq mi.

It is possible to reduce the required area by increasing the amount to pressurize the reservoir. Assuming instead the aquifer average pressure will be elevated by 1000 psi, the required aquifer area is $1371 \mathrm{sq} \mathrm{mi}$, somewhat less than the area of the state of Rhode Island, which has an area of 1545 sq mi. In this case 4 wells will be sufficient.

The minimum aquifer area, assuming pressurization of $1600 \mathrm{psi}$ is approximately $853 \mathrm{sq} \mathrm{mi,} \mathrm{and} 1155$ wells are required.

Of course, greater aquifer thickness reduces the required aquifer area by increasing both injectivity and storability per unit area. If an otherwise similar aquifer is $200 \mathrm{ft}$ thick instead of $100 \mathrm{ft}$, the area required with 1000 psi pressurization is reduced to 686 sq mi, and 2 wells, each requiring a square area approximately $17.5 \mathrm{mi}$ on a side, are sufficient.

\section{General relationships}

Eq. (5) is generalized as follows:

$$
\begin{aligned}
p_{\mathrm{wi}}-p_{\mathrm{i}}= & \Delta p_{\max }=\frac{0.0690 V_{\mathrm{CO}_{2}}}{N_{\mathrm{w}} k h t_{\mathrm{plant}}}\left[\frac{\mu_{\mathrm{CO}_{2}}}{k_{\mathrm{r}, \mathrm{S}_{\mathrm{CO}_{2}}=1}} \ln \left(\frac{r_{\mathrm{dry}}}{r_{\mathrm{w}}}\right)\right. \\
& \left.+\left.\left(\frac{k_{\mathrm{CO}_{2}}}{\mu_{\mathrm{CO}_{2}}}+\frac{k_{\mathrm{rw}}}{\mu_{\mathrm{w}}}\right)^{-1}\right|_{\mathrm{S}_{\mathrm{CO}_{2} \text { avg }}} \ln \left(\frac{r_{\mathrm{BL}}}{r_{\mathrm{dry}}}\right)+\mu_{\mathrm{w}} \ln \left(\frac{0.472}{r_{\mathrm{Bl}}}\right)\right]+\frac{V_{\mathrm{CO}_{2}}}{V_{\mathrm{r} \mathrm{C}_{\mathrm{t}}}}
\end{aligned}
$$

where $\Delta p_{\max }$ is limited to no more than the difference between fracture and hydrostatic pressures, $p_{\mathrm{f}}-p_{\text {hyd }}$, for an aquifer. The pressure of a depleted oil or gas field may be less than hydrostatic. Denoting the term in brackets as $1 / M_{\mathrm{r}}$, this can be further generalized as the following equation:

$$
\frac{\Delta p_{\max }}{V_{\mathrm{CO}_{2}}}=\frac{0.0690}{M_{\mathrm{r}} N_{\mathrm{w}} k h t_{\mathrm{plant}}}+\frac{1}{V_{\mathrm{r}} c_{\mathrm{t}}}
$$

where $N_{\mathrm{w}}$ is the required number of wells. Figs. 1 and 2 show this simple relationship for the specific depths of 4000 and $6000 \mathrm{ft}$ and for injection of 3 million tonnes of $\mathrm{CO}_{2}$ per year. The shallower formation depth has a 


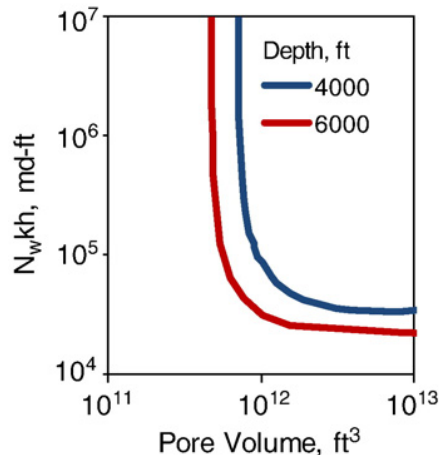

Fig. 1. Relationship between well count, permeability-thickness, and the required minimum pore volume for given relative permeability, $\Delta p_{\max }$, and aquifer depth.

smaller window between formation and fracture pressures, leading to a larger volume requirement.

A critically important message from this generalization concerns storability. The following discussion explains how much pressure matters to the storability in a liquid saturated reservoir.

The volume of fluid that can be stored in a reservoir depends entirely on the fluid compressibility and associated pressure increase, which in turn depends on the reservoir volume. This can be evaluated by starting with the expression for isothermal compressibility.

The isothermal expansibility is defined as

$c=\frac{1}{V}\left(\frac{\partial V}{\partial p}\right)_{T}$

where $V$ is the volume of the fluid. By separation of variables,

$\int_{p_{\mathrm{i}}}^{\bar{p}} c d p=\int_{V_{\mathrm{i}}}^{V} \frac{d V}{V}$

Assuming that $c$ is constant over the pressure range,

$c\left(\bar{p}-p_{\mathrm{i}}\right)=\ln \frac{V}{V_{\mathrm{i}}}$

Rearrangement of Eq. (3) results in

$\frac{V}{V_{\mathrm{i}}}=e^{c\left(\bar{p}-p_{\mathrm{i}}\right)}$

The volume $V$ is equal to $V_{\mathrm{i}}+V_{\mathrm{CO}_{2}}$, that is, the original plus that stored at the higher pressure. Finally, the storability factor, $s_{\mathrm{CO}_{2}}$, is given by

$S_{\mathrm{CO}_{2}}=\frac{V_{\mathrm{CO}_{2}}}{V_{\mathrm{r}}}=e^{c\left(\bar{p}-p_{\mathrm{i}}\right)}-1<e^{c\left(p_{\mathrm{f}}-p_{\mathrm{hyd}}\right)}-1$

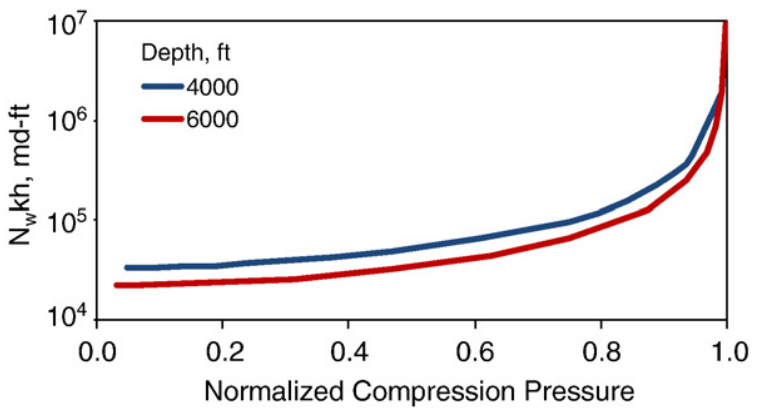

Fig. 2. Relationship between well count, permeability-thickness, and the compression pressure as a fraction of $\Delta p_{\max }$ for given relative permeability, $\Delta p_{\max }$, and aquifer depth.

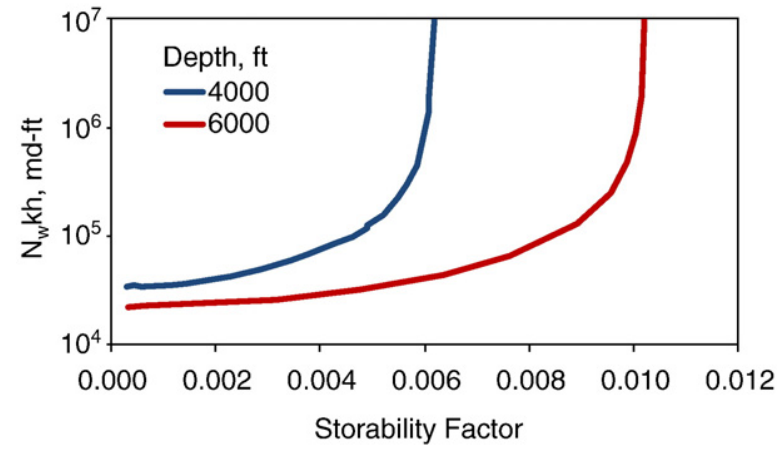

Fig. 3. Relationship between well count, permeability-thickness, and the storability factor for given relative permeability, $\Delta p_{\max }$, and aquifer depth.

Fig. 3 shows the well count $k h$ product as a function of the storability factor. Fig. 3 indicates that the best storability factor is about $1 \%$ of the pore volume. This is in stark contrast to claims in NETL (2007) that suggest that the $\mathrm{CO}_{2}$ storage "efficiency factor between 1 and 4 percent of the bulk volume of saline formations for a 15-85 percent confidence range".

How do we explain this discrepancy, which represents a factor of from 5 to 20? First, NETL (2007) seems to have a typographical error in the above-quoted footnote. The efficiency factor, $E$, is explained in the following equation

$G_{\mathrm{CO}_{2}}=A h \phi \rho_{\mathrm{CO}_{2}} E$

As such, $E$, which is further explained as a product of vertical and areal displacement efficiencies, represents a fraction of the pore volume, not the bulk volume. As such, Fig. 3 is closer to reported storage efficiency, but the upper limit in this estimate corresponds to the lower limit in the NETL estimate.

The remaining discrepancy comes from ignoring the likelihood that injection will be limited by the available volume in the aquifer, as indicated in Fig. 1. The smaller the available pore volume, the more wells will be required, and the more the aquifer pressure must be increased in order to sequester the target volume of $\mathrm{CO}_{2}$.

Figs. 4 and 5 illustrate a fundamental difference between a model with limited aquifer volume and a model for an open aquifer using CMG numerical simulations. With a constant pressure boundary, it is possible to continue injecting $\mathrm{CO}_{2}$ until $\mathrm{CO}_{2}$ breakthrough as long as the injection pressure does not exceed the fracture pressure. For the closed reservoir injection must stop at 30 years to avoid exceeding the fracture pressure constraint. For the open reservoir injection can continue much longer, eventually filling more of the pore space with $\mathrm{CO}_{2}$. Fig. 4 shows the comparison between the bounded and open aquifer cases both in a square drainage area with side $20 \mathrm{mi}$. The character of the pressure profile is similar for the bounded aquifer, but pressure increases with time throughout the aquifer as indicated by the material balance. Fig. 5 shows the same comparison but with distance in the logarithmic scale. This comparison shows that the single phase and two-phase zone radii expand in a similar way for both cases.

\section{Aquifer appraisal}

Results in this work provide insight on what will be required to sequester $\mathrm{CO}_{2}$ from a typical coal power plant. Given aquifer depth, porosity, thickness, permeability, rock compressibility, and relative permeability data along with the brine salinity, the analytical model offers a quick estimate for the required aquifer size for a target total mass of $\mathrm{CO}_{2}$ to be sequestered. Before starting the sequestration, it will be necessary to confirm the aquifer size through an aquifer appraisal process much like the appraisal work done for oil and gas 

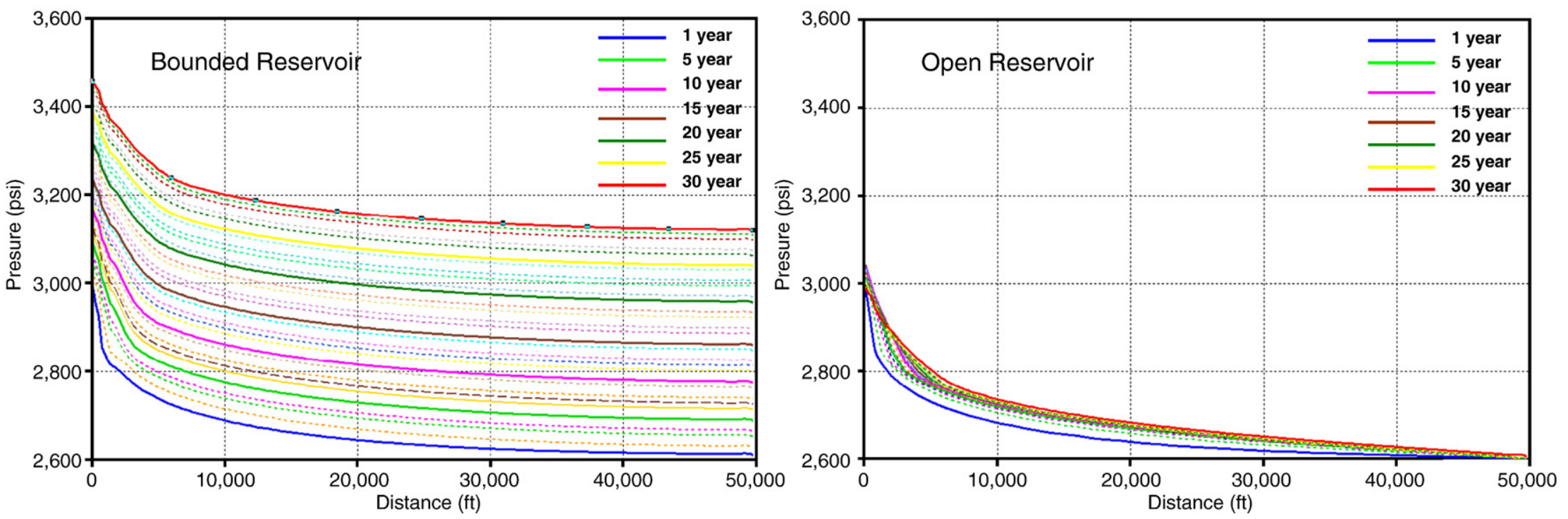

Fig. 4. CMG simulations comparing annual pressure profiles for the bounded and open aquifer cases. Pressure increases with time. 

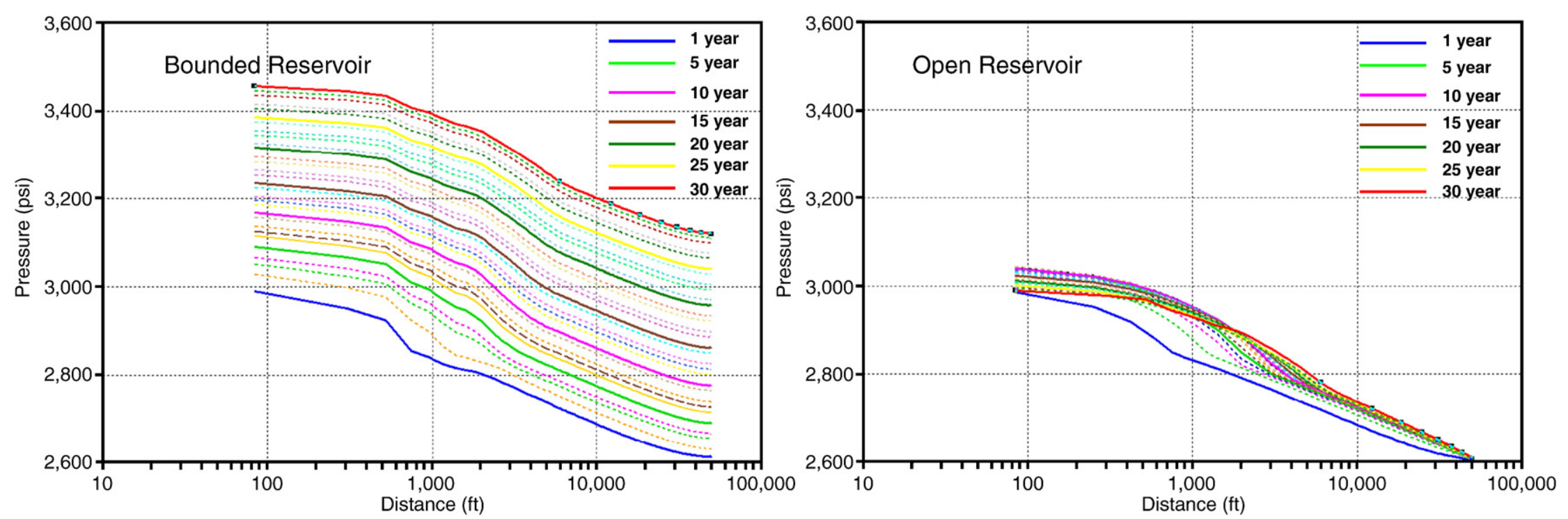

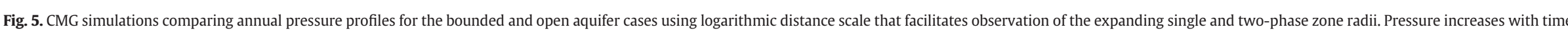


reservoirs. However, a conventional pressure buildup or injection falloff test cannot confirm aquifer areal extent of the size required for a sequestration project because the investigation radius, $r_{\mathrm{i}}$, of a buildup or falloff test is given by

$r_{\mathrm{i}}=\sqrt{\frac{k t}{948 \phi \mu c_{\mathrm{t}}}}$

for $t$ in hours. For porosity and permeability of $20 \%$ and $100 \mathrm{md}$, and compressibility $6 \cdot 10^{-6} \mathrm{psi}^{-1}$, it would take a buildup or falloff duration of 3.6 years to detect aquifer limits at a distance of only ten miles. Alternatively, a pressure buildup or falloff test with 1 month duration will only investigate a radius of about $1.5 \mathrm{mi}$, and not that in reality, because gauge resolution will not be sufficient for such a long time. Additional appraisal wells can be drilled, but it will be difficult to confirm they are in hydraulic communication. Without demonstration of sufficient aquifer areal extent, the project begins with the likely prospect of having to find other aquifers for continued storage of the relentless 79,000 bpd $\mathrm{CO}_{2}$.

\section{Conclusions}

The implications of this work are profound. A simple analytical model shows immediate results very similar to those that take hours to produce with numerical simulation. Much more important, the work shows that models that assume a constant pressure outer boundary for reservoirs intended for $\mathrm{CO}_{2}$ sequestration are missing the critical point that the reservoir pressure will build up under injection at constant rate. Instead of the $1-4 \%$ of bulk volume storability factor indicated prominently in the literature, which is based on erroneous steady state modeling, our finding is that $\mathrm{CO}_{2}$ can occupy no more than $1 \%$ of the pore volume and likely as much as 100 times less.

This work has related the volume of the reservoir that would be adequate to store $\mathrm{CO}_{2}$ with the need to sustain injectivity. The two are intimately connected. In applying this to a commercial power plant the findings suggest that for a small number of wells the areal extent of the reservoir would be enormous, the size of a small US state. Conversely, for more moderate size reservoirs, still the size of Alaska's Prudhoe Bay reservoir, and with moderate permeability there would be a need for hundreds of wells. Neither of these bodes well for geological $\mathrm{CO}_{2}$ sequestration and the findings of this work clearly suggest that it is not a practical means to provide any substantive reduction in $\mathrm{CO}_{2}$ emissions, although it has been repeatedly presented as such by others.

\section{Nomenclature}

A areal extent, sq $\mathrm{ft}$

$c_{\mathrm{t}} \quad$ total compressibility at the end of injection, $\mathrm{psi}^{-1}$

$c_{\mathrm{ti}} \quad$ initial total compressibility, $\mathrm{psi}^{-1}$

$E \quad$ displacement efficiency factor, dimensionless

$\mathrm{G}_{\mathrm{CO}_{2}} \quad \mathrm{CO}_{2}$ pore volume, $\mathrm{cu} \mathrm{ft}$

$h$ reservoir thickness, $\mathrm{ft}$

$H \quad$ depth, $\mathrm{ft}$

$k$ permeability, md

$k_{\mathrm{r}} \quad$ relative permeability, dimensionless

$p_{\mathrm{i}} \quad$ initial reservoir pressure, psi

$p_{\text {wi }} \quad$ bottomhole injection pressure, psi

$q \quad$ injection rate, STB/d

$r_{\mathrm{i}} \quad$ pressure transient test radius of investigation, $\mathrm{ft}$

$r_{\mathrm{w}} \quad$ wellbore radius, $\mathrm{ft}$

$\mathrm{S}_{\mathrm{CO}_{2}} \quad$ storability factor, dimensionless

$\mathrm{S}_{\mathrm{CO}_{2}} \quad$ gas saturation, dimensionless
$S_{\mathrm{w}} \quad$ water saturation, dimensionless

$t \quad$ time, h

$t_{\text {plant }}$ duration of $\mathrm{CO}_{2}$ injection, $\mathrm{yr}$

$V_{\mathrm{CO}_{2}} \quad \mathrm{CO}_{2}$ volume to inject, $\mathrm{cu} \mathrm{ft}$

$V_{\mathrm{r}} \quad$ reservoir volume, $\mathrm{cu} \mathrm{ft}$

Symbols

$\phi \quad$ porosity, dimensionless

$\mu \quad$ viscosity, $\mathrm{cp}$

\section{Acknowledgement}

The authors offer special thanks to Abhishek Anchliya for considerable help in ensuring the accuracy of the analytical solutions as compared to CMG numerical simulations.

\section{References}

Baklid, A., Korbo, R., 1996. Sleipner Vest $\mathrm{CO}_{2}$ disposal, $\mathrm{CO}_{2}$ injection into a shallow underground aquifer. Paper SPE 36600.

Bickle, M., Chadwick, A., Huppert, H.E., Hallworth, M., Lyle, S., 2007. Modelling carbon dioxide accumulation at Sleipner: implications for underground carbon storage. EPSL 255, 164-176.

Buckley, S.E., Leverett, M.C., 1942. Mechanism of fluid displacement in sands. Pet Trans., AIME 146, 107-116.

Burton, M., Kumar, N., Bryant, S.L., 2008. Time dependent injectivity during $\mathrm{CO}_{2}$ storage in aquifers. SPE 113937 presented at the SPE/DOE Improved Oil Recovery Symposium, Tulsa, OK.

Economides, M.J., Nolte, K.G., 2000. Reservoir Stimulation, Third Edition. Wiley, NY 750 pp. (hardbound).

Ennis-King, J., Paterson, L., 2002. Engineering aspects of geological sequestration of carbon dioxide. Paper SPE 77809.

Evans, T., Melzer, L.S., 2009. North American $\mathrm{CO}_{2}$ status. Presented at the Enhanced Oil Recovery Institute's Third Annual $\mathrm{CO}_{2}$ Conference.

House, N.J., Faulder, D.D., Olson, G.L., Fanchi, J.R., 2003. Simulation study of $\mathrm{CO}_{2}$ sequestration in a North Sea formation. Paper SPE 81202.

IPCC, 2007. Climate Change 2007: the physical science basis. In: Solomon, S., Qin, D. Manning, M., Chen, Z., Marquis, M., Averyt, K.B., Tignor, M., Miller, H.L. (Eds.), Contribution of Working Group I to the Fourth Assessment Report of the Intergovernmental Panel on Climate Change. Cambridge University Press, Cambridge. http://www.ipcc.ch/pdf/assessment-report/ar4/wg1/ar4-wg1-faqs.pdf, 2007.

Izpec, O., Demiral, B., Bertin, H., Akin, S., 2006. Experimental and numerical modeling of direct injection of $\mathrm{CO}_{2}$ into carbonate formations. Paper SPE 100809.

Jarrell, P.M., Fox, C.E., Stein, M.H., Webb, S.L., 2002. Practical aspects of $\mathrm{CO}_{2}$ flooding. SPE Monogr. Ser. 22

Kumar, A., Ozah, R., Noh, M., Pope, G.A., Bryant, S., Sepehrnoori, K., Lake, L.W., September 2005. Reservoir simulation of $\mathrm{CO}_{2}$ storage in deep saline aquifers. SPEJ 336-348.

NETL, March 2007. Carbon Sequestration Atlas of the United States and Canada. U.S. Department of Energy, Office of Fossil Energy, National Energy Technology Laboratory.

Nghiem, L., Sammon, P., Grabenstetter, J., Okhuma, H., 2004. Modeling $\mathrm{CO}_{2}$ storage in aquifers with a fully-coupled geochemical EOS compositional simulator. Paper SPE 89474.

Noh, M., Lake, L.W., Bryant, S.L., Arague-Martinez, A., 2004. Implications of coupling fractional flow and geochemistry for $\mathrm{CO}_{2}$ injection in aquifers. Paper SPE 89341.

Orr, F.M., September 2004. Storage of carbon dioxide in geologic formations. JPT 90-97.

Pruess, K., June 2004. Numerical simulation of $\mathrm{CO}_{2}$ leakage from a geologic disposal reservoir, including transitions form super-to-subcritical conditions, and boiling of liquid $\mathrm{CO}_{2}$. SPEJ 237-248.

Pruess, K., Xu, T., Apps, J., Garcia, J., 2001. Numerical modeling of aquifer disposal of $\mathrm{CO}_{2}$. Paper SPE 66537.

Schembre-McCabe, J.M., Kamath, J., Gurton, R., 2007. Mechanistic studies of $\mathrm{CO}_{2}$ sequestration. IPTC 11391.

Sengul, M., 2006. $\mathrm{CO}_{2}$ sequestration - a safe transition technology. Paper SPE 98617.

van der Meer, L.G.H, van Wees, J.D., 2006. Limitations to storage pressure in finite saline aquifers, and the effect of $\mathrm{CO}_{2}$ solubility on storage pressure. SPE 103342.

van Engelenburg, B.C.W, Blok, K., 1993. Disposal of carbon dioxide in permeable underground layers: a feasible option? Clim. Change 23, 55-68.

Zakrisson, J., Edman, I., Cinar, Y., 2008. Multiwell injectivity for $\mathrm{CO}_{2}$ storage. SPE 116355 Presented at the Asia Pacific Oil and Gas Conference and Exhibition, Perth, Australia. 